\title{
Evolution of Spatial Pattern and Influencing Factors of Population in Guangdong-Hong Kong-Macao Greater Bay Area
}

\author{
Hegang Yao \\ School of Economics, Economic Research Institute on Special Economic Zone, Jinan University, Guangzhou, China \\ Email: yaohegang123@163.com
}

How to cite this paper: Yao, H. G. (2020). Evolution of Spatial Pattern and Influencing Factors of Population in Guangdong-Hong Kong-Macao Greater Bay Area. Open Journal of Social Sciences, 8, 33-46. https://doi.org/10.4236/jss.2020.84003

Received: March 6, 2020

Accepted: March 29, 2020

Published: April 2, 2020

Copyright $\odot 2020$ by author(s) and Scientific Research Publishing Inc. This work is licensed under the Creative Commons Attribution International License (CC BY 4.0).

http://creativecommons.org/licenses/by/4.0/

\begin{abstract}
This paper takes the population of Guangdong-Hong Kong-Macao Greater Bay Area as the research object, and analyzes the evolution of population spatial pattern of Guangdong-Hong Kong-Macao Greater Bay area. According to the data, eleven areas of "two districts and nine cities" in Guangdong-Hong Kong-Macao Greater Bay area district are divided into three levels; the relative migration index is used to divide them into relative moving in areas and relative moving out areas, on the other hand, using the spatial panel data regression model to analyze the influencing factors of the evolution of the spatial pattern of population in Guangdong, Hong Kong and Macao. The main conclusions are as follows: 1) from 2005 to 2017, the spatial distribution of population in Guangdong-Hong Kong-Macao Greater Bay area shows a trend of continuous aggregation. The geographic concentration of population in Shenzhen, Dongguan, Hong Kong and Macao is relatively high. Guangzhou, Foshan, Shenzhen and Zhongshan belong to the region of relative migration. 2) This paper selects the quality of basic education, housing price, industrial structure, medical service level, traffic convenience and income level as independent variables, adds the spatial weight matrix, and uses the spatial error model to regress the geographic concentration of population. Main results: income level, industrial structure, medical condition and traffic convenience level all promote the population agglomeration of Guangdong-Hong Kong-Macao Greater Bay area.
\end{abstract}

\section{Keywords}

Guangdong-Hong Kong-Macao Greater Bay Area, Geographical Concentration, Influencing Factors, Spatial Distribution, Population 


\section{Introduction}

\subsection{Research Background}

The spatial distribution of population refers to the geographical distribution of population in a specific time section, which is the result of the interaction of human and nature, society, politics, economy and other factors. The spatial distribution of population and its changes are the key issues in the development of regional economy, and also the important basis for the formulation of major regional development strategic planning. Guangdong-Hong Kong-Macao Greater Bay Area officially entered the national strategic level in 2017, including two special administrative regions of Hong Kong and Macao and nine cities of Guangzhou, Shenzhen, Zhuhai, Foshan, Zhongshan, Dongguan, Huizhou and Jiangmen, covering an area of 56,000 square kilometers, with a population of more than 69 million and a GDP of more than 10 trillion yuan in 2017. It is an important space for China to build a world-class city group and participate in global competition Intermediate carrier. "Nine cities in the Pearl River Delta", the main component of Guangdong-Hong Kong-Macao and Greater Bay area, has been one of the regions attracting the most foreign population since the reform and opening up. The implementation of the development strategy of Guangdong-Hong Kong-Macao Greater Bay area will definitely further attract more foreign people, and the population will gather in a large number in a certain area, which will promote the rapid development of economy and other aspects, but also bring a series of problems such as environmental pollution, traffic congestion, housing shortage, and medical stress. Therefore, it is of great theoretical and practical significance to study the evolution characteristics and influencing factors of the spatial distribution of population in Guangdong-Hong Kong-Macao Greater Bay area for the optimization of regional industries, the rational allocation of resources and the management of population regulation in the future.

\subsection{Literature Review}

Domestic scholars mainly study the population structure, density change and spatial distribution of counties, cities, provinces, urban agglomerations and national regions. For example, Chunmeng Du used the population and built-up area data of 285 prefecture level cities in China from 2006 to 2016 to study the population density change and spatial distribution and explored the influencing factors of population density change ( $\mathrm{Du}$, Jiao, \& Xu, 2018). Many scholars have studied the provincial regions of China (Hunan, Shanxi, etc.) and large-scale urban agglomerations (Yangtze River Delta, Beijing Tianjin Hebei, Pearl River Delta and Eastern three provinces, etc.) in terms of population temporal and spatial evolution pattern and driving factors (Wang \& Qin, 2013; Mu, 2014; Deng, Li, \& Bin, 2017; Yu, Song, Hao, \& Rong, 2017); some scholars put the population of a single provincial capital city and some typical cities (Wuhan, Shanghai, Guangzhou, etc.). Distribution is studied in combination with local 
industry, economy, politics, science and Technology (Yu, Liu, \& Yang, 2012; Zhou, Tu, Lu, Yan, \& Wu, 2011). Generally speaking, the main reasons for the change of population density in time and space include economic development level, urban scale, location factors, urban development policies, etc., which can also be subdivided into education, medical treatment, transportation, income level, etc. each city or region has its own unique historical characteristics and modern development characteristics, which is also the value of scholars' research.

Many scholars have analyzed the evolution of spatial distribution of population and its influencing factors in Guangdong-Hong Kong-Macao Greater Bay area and its typical internal areas. Zhen You analyzed the spatial distribution pattern of population and its changing characteristics in the Pearl River Delta in the past 30 years by using the data of the four population censuses from 1982 to 2010; Jin Liu explored the interaction mechanism among industry, population and space in the Pearl River Delta urban agglomeration; Zhiwei Du dynamically observed the time change of population contraction in different types of cities in Guangdong Province And spatial distribution, industrial power, regional development differences, and local financial strength are the main factors of urban population change; Yaxi Feng described the changes in the number and spatial distribution of Guangzhou population in the past 30 years, and analyzed the relation-ship between the spatial distribution of population and economic structure from the perspective of employment structure, output value composition, regional eco-nomic characteristics (You, Wang, Feng, \& Yang, 2013; Liu \& Tian, 2018; Du, Zhang, Ye, Jin, \& Wang, 2019).

\subsection{Purpose and Significance of Research}

This paper intends to describe the population concentration of "two districts and nine cities" in Guangdong-Hong Kong-Macao Greater Bay area by using GIS spatial measurement software, and analyze the relationship between population density and education, income level, housing price, medical treatment, transportation and industrial structure by using data processing software Stata, so as to understand the spatial distribution of population among various parts of Guangdong-Hong Kong-Macao Greater Bay area and to serve the future of Guangdong, Hong Kong and Macao. There are some useful suggestions for the construction of the Bay area.

\section{Research Methods and Data Sources}

\subsection{Research Method}

Geographic concentration of population. The index that measures the concentration degree of population elements in a certain region. It can not only reflect the distribution of population in time, but also reflect the position and role of a region in the same region or the whole. The calculation formula is:

$$
R_{i}\left(\operatorname{Pop}_{i}\right)=\frac{\mathrm{Pop}_{i}}{\mathrm{Acr}_{i}} / \frac{\sum \mathrm{Pop}_{i}}{\sum \mathrm{Acr}_{\mathrm{i}}}
$$


where: $R_{i}\left(\mathrm{Pop}_{i}\right)$ represents the geographical concentration index of population in $I$ region in a certain year; [Pop] $I$ and Acr I represent the population and land area of $I$ region respectively; $\Sigma$ represents the sum of relevant attribute values in the research area.

Population relative migration index. The actual response is the ratio of the average development speed of a unit population in the region to the average development speed of the regional population. In order to further clearly show the relative changes of population in each district and city, this paper uses Naiquan Liu and others to construct the relative migration coefficient based on the relationship between the geometric mean of the geographic concentration of population and 1 (Liu \& Geng, 2015). The calculation formula is as follows:

$$
T_{P}=\left(R_{i T} / R_{i 1}\right)^{\frac{1}{T-1}}-1
$$

where: $T_{P}$ represents the relative migration coefficient of population in each unit of the region; $R_{i 1}$ and $R_{i T}$ represent the geographic concentration of population in the base period and reporting period of unit $I$ of the region respectively. When $T_{p}<0$, the population of unit $I$ in this region shows relative migration; when $T_{p}=0$, the population of unit $I$ in this region shows relative status quo; when $T_{p}>0$, the population of unit $I$ in this region shows relative migration.

ESDA. The technology of exploratory spatial data analysis (ESDA) is the core of spatial statistical analysis. It aims to describe and display the spatial distribution of objects, and reveal spatial relationships, clusters and other heterogeneous spatial patterns. In this paper, ArcGIS spatial measurement software will be used to analyze spatial dependence and spatial heterogeneity of population spatial distribution in Guangdong-Hong Kong-Macao Greater Bay Area by using global Moran's I and local indicators of Spatial Association (LISA). Moran's I:

$$
I=\frac{\sum_{i} \sum_{j}\left(X_{i}-\bar{X}\right)\left(X_{j}-\bar{X}\right)}{s^{2} \sum_{i} \sum_{j} W_{i j}}
$$

where: $X_{i}$ is the observation value of area $i ; S^{2}=\sum\left(X_{i}-\bar{X}\right)^{2} / n ; \quad W_{i j}$ is the spatial weight value.

Local spatial autocorrelation: reveals the autocorrelation of local element attributes in adjacent spaces. Local Moran's I calculation formula:

$$
I_{i}=Z_{i} \sum_{i}^{n} W_{i j} Z_{i j}
$$

In the formula, $Z_{i}, Z_{j}$ are the standardized forms of observation values; $I$ and $j$ represent different regional units; $W_{i j}$ represents any element of spatial weight matrix to define the mutual adjacency relationship of spatial objects.

\subsection{Data Sources}

The relevant data used in this article mainly come from the Guangdong Statis- 
tical Yearbook, Hong Kong Statistical Yearbook, and Macau Statistical Yearbook from 2005 to 2018. The data includes the population, administrative area, GDP, income level, highway mileage, etc. of each district and city in the Guangdong-Hong Kong Greater Bay Area. Considering the types of data required for setting the data model in this article and the completeness and continuity of data published by the districts and cities, the data time span used in this article is 2005-2017. In the selection of cross-section data, the data statistics of Shunde from 2010 to 2016 were independent, so the data in this interval was merged into Foshan. In addition, there are some problems such as missing data or inconsistent statistical calibers, etc. The data have been amended to some extent through comparison with data from the National Economic and Social Development Statistical Bulletin issued by multi-level government departments.

\section{Spatial and Temporal Distribution of Population in Guangdong, Hong Kong and Macao Greater Bay area}

\subsection{Geographical Distribution and Basic Situation of Guangdong-Hong Kong-Macao Greater Bay Area}

Guangdong-Hong Kong-Macao Greater Bay area, one of the first open areas in China, is the economic hinterland of the "Great Pearl River Delta region". It is also a part of the "one belt, one road" project. It plays an important role in supporting national economic development and opening to the outside world, Guangdong-Hong Kong-Macao Greater Bay area includes two special administrative regions of Hong Kong and Macao and nine cities of Guangzhou (GZ), Shenzhen (SZ), Zhuhai (ZH), Foshan (FS), Huizhou (HZ), Zhongshan (ZS), Dongguan (DG), and Jiangmen (JM), as shown in Figure 1.

\subsection{Geographic Concentration of Population}

In order to make a clear comparative analysis of the changes in the population aggregation layout of Guangdong-Hong Kong-Macao Greater Bay area, 2005, 2011 and 2017 are selected as representative years to divide the geographic concentration of population in each district and city of Guangdong-Hong Kong-Macao Greater Bay area into three levels: the first level area is lower than 0.25 times the average value; the second level area is 0.25 - 0.50 times the average value; the third level area is higher than 0.5 times the average value; according to the relative migration coefficient of population The districts and cities in Guangdong-Hong Kong-Macao are divided into two parts: the relative moving in area and the relative moving out area. Use Excel software to make the diagram, and the relevant results are shown in Table 1.

According to the above classification method, Guangdong-Hong Kong-Macao Greater Bay area can be divided into the first level areas including Hong Kong, Macao, Shenzhen and Dongguan; the second level areas are Guangzhou, Zhuhai and Zhaoqing; the third level areas are Huizhou, Zhongshan and Jiangmen. On 


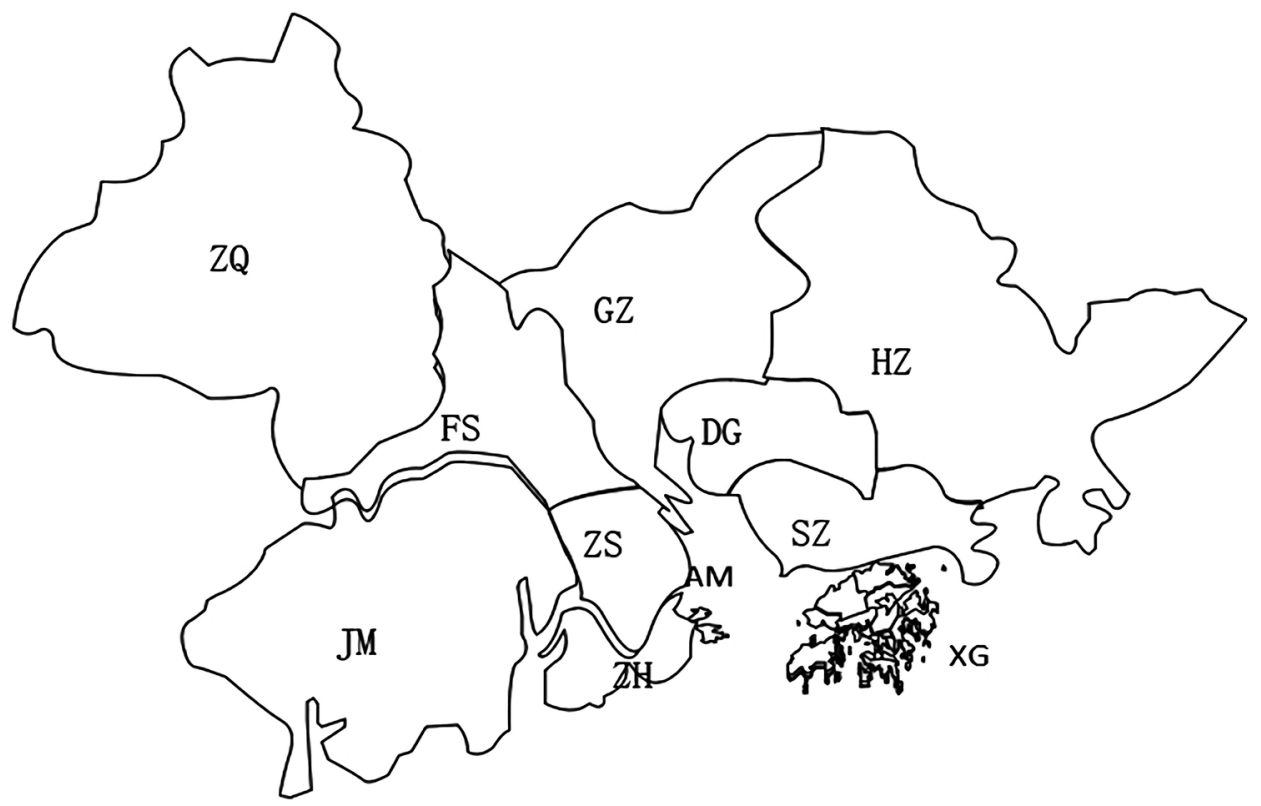

Figure 1. Map of Guangdong-Hong Kong-Macao Greater Bay Area.

Table 1. Map of Guangdong-Hong Kong-Macao Greater Bay Area.

\begin{tabular}{cccccccc}
\hline & \multicolumn{2}{c}{2005} & \multicolumn{2}{c}{2011} & \multicolumn{2}{c}{2017} & $2017-2005$ \\
\hline & GPR & $T$ & GPR & $T$ & GPR & $T$ & $T_{p}$ \\
\hline GZ & 1.346 & 0.381 & 1.513 & 0.455 & 1.570 & 0.464 & 0.014 \\
SZ & 4.468 & 1.267 & 4.636 & 1.394 & 5.052 & 1.493 & 0.011 \\
ZH & 0.921 & 0.261 & 0.808 & 0.243 & 0.820 & 0.242 & -0.010 \\
FS & 1.588 & 0.450 & 1.679 & 0.505 & 1.623 & 0.480 & 0.001 \\
HZ & 0.350 & 0.099 & 0.360 & 0.108 & 0.339 & 0.100 & -0.002 \\
DG & 2.804 & 0.795 & 2.960 & 0.890 & 2.731 & 0.807 & -0.002 \\
ZS & 1.425 & 0.404 & 1.540 & 0.463 & 1.471 & 0.435 & 0.002 \\
JM & 0.453 & 0.128 & 0.414 & 0.124 & 0.386 & 0.114 & -0.014 \\
ZQ & 0.268 & 0.076 & 0.225 & 0.067 & 0.222 & 0.065 & -0.016 \\
XG & 6.915 & 1.962 & 5.974 & 1.797 & 5.686 & 1.681 & -0.017 \\
AM & 18.22 & 5.171 & 16.44 & 4.948 & 17.30 & 5.115 & -0.004 \\
\hline
\end{tabular}

Note: Trefers to the comparison between GPR and the average value of the year.

the whole, the population changes of each district and city in Guangdong-Hong Kong-Macao Greater Bay area are relatively stable, among which, Foshan was upgraded from the second level district to the first level district in 2005-2011, and from the first level district to the second level district in 2011-2017, and the classification of other regions has not changed.

Figure 2 shows the population relative migration index distribution of each district and city in Guangdong-Hong Kong-Macao Greater Bay area from 2005 


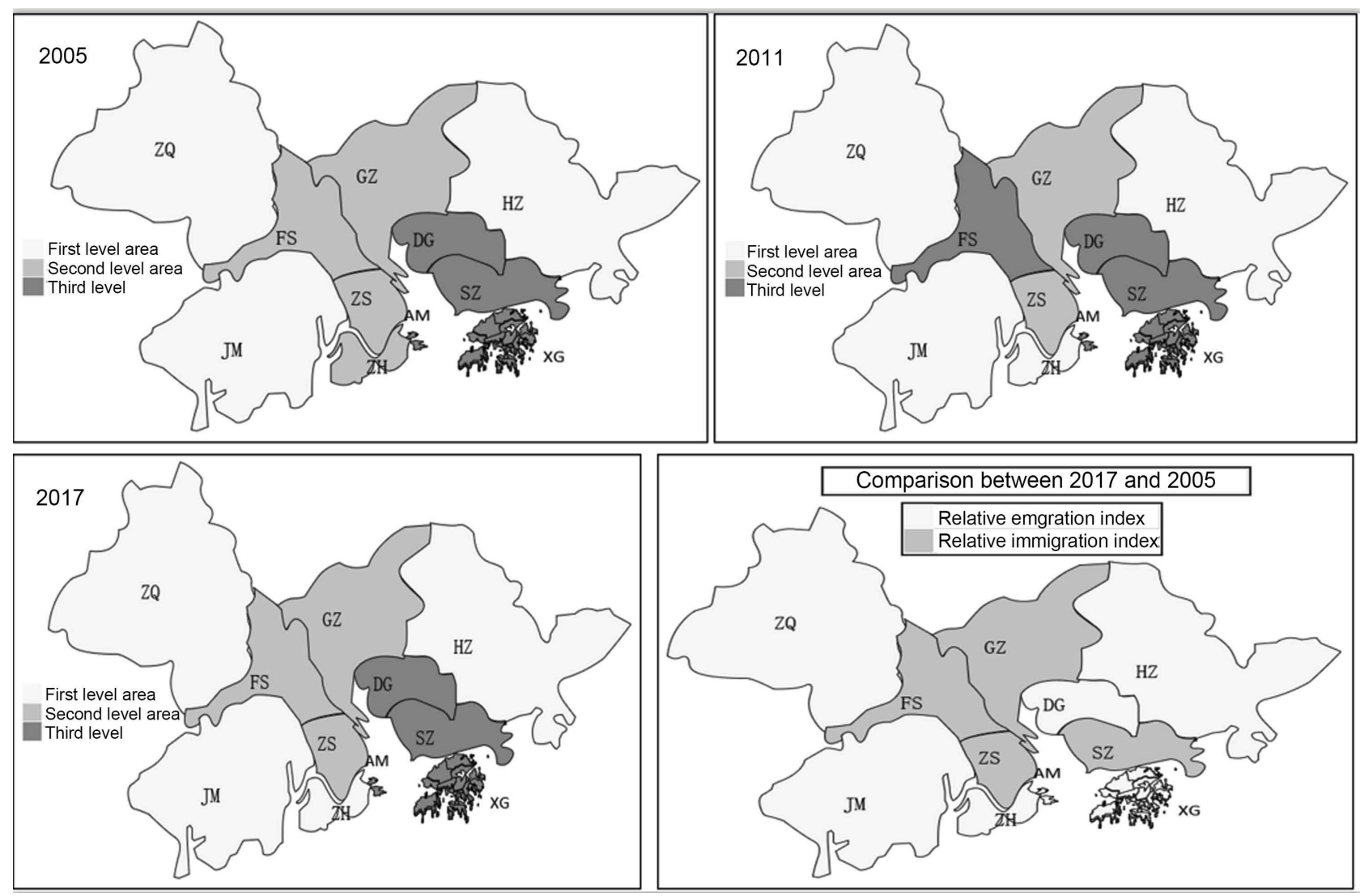

Figure 2. Population aggregation change and relative change trend of districts and cities in Guangdong-Hong Kong-Macao Greater Bay area from 2005 to 2017.

to 2017. Guangzhou, Foshan, Shenzhen and Zhongshan belong to the relative migration area, while Huizhou, Zhaoqing, Foshan, Jiangmen, Zhuhai, Dongguan, Hong Kong and Macao belong to the relative migration area.

From 2005 to 2017, the population density of Guangdong-Hong Kong-Macao Greater Bay area increased significantly, the geographic concentration of population increased significantly, and the population distribution of each district and city was relatively stable. What factors have led to the population growth and the change of population aggregation layout in the region of Guangdong, Hong Kong and Macao? This paper will use panel data for empirical research.

\section{Data Interpretation, Model Setting and Processing Methods}

\subsection{Model Setting}

Many scholars have studied the change of population spatial aggregation and its influencing factors (Meng \& Xin, 2018; Shen \& Luo, 2019). Based on the research of the above scholars, this paper selects industrial structure, house price, income, transportation convenience, medical service level and basic education quality as the influencing factors of population aggregation change, and constructs panel data model, which is as follows 


$$
\begin{aligned}
\text { gpr }_{i t}= & \beta_{0}+\beta_{1} \text { inds }_{i t}++\beta_{3} \text { incom }_{i t}+\beta_{4} \text { traf }_{i t}+\beta_{5} \text { rtt }_{i t} \\
& +\beta_{6} \mathrm{hp}_{i t}+\beta_{7} \text { medi }_{i t}+\rho \sum_{j} \omega_{i j} \text { gpr }_{j t}+\varepsilon_{i t}
\end{aligned}
$$

In the model, gpr is the dependent variable, which is used to reflect the degree of spatial aggregation of population; inds is used to measure the factors of industrial structure; incom is used to measure the level of income; $\mathrm{rtt}$ is used to measure the quality of basic education; medi is used to measure the medical and health conditions; HP is used to measure the price of housing; $i$ and $t$ are used to represent the city and time respectively; $\beta_{0}, \beta_{1}, \cdots, \beta_{7}$ are the coefficients to be estimated of independent variables; $\varepsilon$ is the random disturbance term. $\rho$ is the spatial regression coefficient, $\omega_{i j}$ is the weight matrix $W$;

\subsection{Data Interpretation and Processing Methods}

Industrial structure (inds). The spatial distribution of urban population is the result of various social and economic activities, and the concentration and development of industry is the direct cause of the change of spatial distribution of population. As one of the engines of China's economic development, the development of the secondary and tertiary industries plays an important strategic role in the social economy. However, with the rapid development of the city, it is gradually changing its strategic positioning, which also has a significant impact on the distribution and proportion of the secondary and tertiary industries in the city, further affecting the distribution and change of the population; meanwhile, the Ministry The establishment of hi-tech industrial development zones in the relevant suburbs will also guide part of the population to flow to the suburbs. Therefore, the second industry represented by industrial enterprises and the third industry represented by financial services will inevitably lead to the change of population distribution in different regions. Therefore, in this data model, the GDP added value of the tertiary industry is used to measure the industrial structure of Guangdong-Hong Kong-Macao Greater Bay area.

Income level. As we all know, people living and working in different cities will have great differences in their living expenses and incomes, such as the daily necessities, firewood, rice, oil and salt. From the perspective of economic utility theory, each individual will rationally maximize their own utility. Under the constraints of living costs, they will choose the best living location for themselves and pursue the highest income. With the improvement of income conditions, people with high education and high technology will make relevant choices in pursuit of higher quality of life and higher survival status. Therefore, the income level of different regions will certainly have a certain impact on population distribution.

Traffic convenience (traf). The convenience of transportation and logistics in different regions is one of the decisive forces of the local economic development, which further affects the time cost and living cost of the employed population. It can be seen that the degree of convenient transportation will inevitably affect the employment population and the residential location choice of commuter popu- 
lation. The ratio of the length of roads to the area of each area in Guangdong, Hong Kong and Macao is selected to measure the level of traffic convenience.

Quality of basic education (rtt). The uneven distribution of basic education resources has always been a major academic topic of concern to scholars. At present, there is no good measure to significantly improve this situation. In the case of not significantly changing the status quo, it is an important goal for people to pursue high-quality basic education level and choose rich regional residence and education of basic education resources. It can be seen that the distribution of basic education resources will inevitably have a significant impact on the spatial distribution of population. Therefore, this paper uses the teacher-student ratio of primary and secondary schools to reflect the quality of basic education in nine cities, two districts, Guangdong-Hong Kong-Macao Greater Bay area.

Medical and health service level (medi). The medical and health resources are mainly distributed in the central urban areas of big cities. Compared with the central urban areas, the medical and health service conditions, medical insurance system guarantee and the convenience of medical treatment in other suburbs, small cities and rural areas are different. It is also reasonable that the difference of medical service level in different regions has an impact on the spatial distribution of population, so this paper uses the proportion of the number of hospital beds in the population to measure the difference of medical resource level in different regions of Guangdong, Hong Kong and Macao.

House price (hp). Nowadays, the important position of real estate in Chinese people's life and work need not be described too much. The house price in China has almost never stopped rising in the past decade, which has a huge impact on people's life. The amount of personal wealth and continuous income are different. The land policies in various regions are different, and the number of land available for real estate development is very large Difference, at the same time, the quality of a series of public infrastructure around the housing is different, as well as the demand of the permanent residents in different regions for housing and rental housing, which has different impact on the change trend of housing prices in different regions, and the rising housing prices in turn affect people's demand in all aspects. For example, the better welfare facilities in the central city attract more foreign population, which aggravates the scarcity of high-quality resources. The house price will rise accordingly, and the rise of house price will also to some extent exclude excessive population aggregation. It can be seen that the level of housing prices in different regions will also have some impact on the spatial distribution of the population. Therefore, this paper uses the average transaction price of commercial residential housing to measure the housing price level of all districts and cities in Guangdong-Hong Kong-Macao Greater Bay area.

\section{Empirical Analysis of the Factors Influencing the Spatial Distribution of Population in the Guangdong-Hong Kong-Macao Greater Bay Area}

Three main methods are used in the analysis of spatial panel model: maximum 
likelihood method (MLE), generalized moment estimation method (GMM) and two-stage least square method (2SLS). The maximum likelihood method is most commonly used. In this paper, MLE and Stata are used to estimate the model parameters.

\subsection{Model Setting Test}

The test values of the five spatial correlation tests in Table 2 are not statistically significant except for robot LMlag, and the others are significant at the level of $10 \%$, which indicates that the population distribution of Guangdong-Hong Kong-Macao Greater Bay area really conforms to certain spatial correlation characteristics. Therefore, the spatial econometric model adopted in the data analysis in this paper is relatively reasonable. It can be seen from the table that LMerr and robot LMerr are superior to LMlag and robot LMlag respectively in the significant level, and only the statistical value of LMerr is significant at the level of about $5 \%$. According to the criteria proposed by anselin, it shows that the spatial error model is more suitable for data analysis in this paper than the spatial lag model.

Moran I was used to test the spatial correlation of population distribution in Guangdong-Hong Kong-Macao Greater Bay area. The statistical value of Moran $I$ is between -1 and 1 . When it is less than 0 , it can be considered that there is spatial exclusion in population distribution of each district and city; when it is greater than 0 , it can be considered that there is spatial aggregation. The statistical value of Moran I in Table 2 is 0.168 , which is greater than 0 . Therefore, it can be seen that there is a relatively consistent population aggregation between adjacent districts and cities in Guangdong-Hong Kong-Macao Greater Bay area.

\subsection{Empirical Results and Analysis}

This paper shows the estimation results of the spatial error model (sepdm). In order to observe the change trend of the estimated values of the parameters of each variable and verify the robustness of the estimation results, this paper uses the method of gradually increasing variables for regression to show the results, as shown in Table 3.

According to the regression results of the eight models shown in Table 3, model 6 is superior to other models in terms of goodness of fit. It can be seen

Table 2. Model setting test results.

\begin{tabular}{cccccc}
\hline Test method & Inspection items & sample size & Test value & $P$ value & conclusion \\
\hline Moran I & Spatial autocorrelation & 72 & 0.168 & 0.021 & exist \\
LMlag & Spatial autocorrelation & 72 & 2.455 & 0.082 & exist \\
LMerr & Spatial autocorrelation & 72 & 5.628 & 0.053 & exist \\
Robust LMlag & Spatial autocorrelation & 72 & 3.050 & 0.186 & not exist \\
Robust LMerr & Spatial autocorrelation & 72 & 1.363 & 0.068 & exist \\
\hline
\end{tabular}


Table 3. Empirical analysis of spatial distribution of population in Guangdong-Hong Kong-Macao Greater Bay Area.

\begin{tabular}{|c|c|c|c|c|c|c|c|c|}
\hline variable & mode1 & model2 & mode3 & model4 & model5 & model6 & model7 & model8 \\
\hline \multirow[t]{2}{*}{ inds } & $19.839^{* * *}$ & $20.291^{* * *}$ & $18.697^{\star *}$ & $19.051^{* * *}$ & $17.562^{\star *}$ & $16.435^{\star * *}$ & $14.382^{* * *}$ & $10.471^{\star}$ \\
\hline & 1.134 & 0.989 & 1.208 & 0.879 & 1.037 & 1.112 & 1.544 & 2.675 \\
\hline \multirow[t]{2}{*}{ income } & & $1.658^{\star * *}$ & $1.711^{\star * *}$ & $1.766^{\star *}$ & $1.806^{* * *}$ & $1.823^{\star * *}$ & $1.654^{* * *}$ & $1.874^{* * *}$ \\
\hline & & 0.154 & 0.108 & 0.148 & 0.170 & 0.162 & 0.159 & 2.145 \\
\hline \multirow[t]{2}{*}{ traf } & & & $0.036^{* * *}$ & $0.078^{\star * \star}$ & $0.054^{\star \star *}$ & $0.063^{* * *}$ & $0.069^{* * *}$ & $0.087^{\star *}$ \\
\hline & & & 0.026 & 0.025 & 0.025 & 0.025 & 0.027 & 0.046 \\
\hline \multirow[t]{2}{*}{$\mathrm{rtt}$} & & & & $-8.054^{\star \star \star}$ & $-6.857^{\star * *}$ & $-5.679^{\star * *}$ & $-7.473^{* * *}$ & $-10.076^{* * x}$ \\
\hline & & & & 11.046 & 10.989 & 12.866 & 10.967 & 20.354 \\
\hline \multirow[t]{2}{*}{ medi } & & & & & $0.252^{* * *}$ & $0.238^{* * *}$ & $0.234^{* * *}$ & $0.445^{\star *}$ \\
\hline & & & & & -0.036 & -0.045 & 0.043 & -0.857 \\
\hline \multirow[t]{2}{*}{ hp } & & & & & & $0.116^{* *}$ & $0.143^{* * *}$ & $0.285^{\star * *}$ \\
\hline & & & & & & -0.048 & -0.067 & -1.324 \\
\hline R2 & 0.805 & 0.826 & 0.835 & 0.841 & 0.865 & 0.905 & 0.864 & 0.835 \\
\hline Model & SEPDM & SEPDM & SEPDM & SEPDM & SEPDM & SEPDM & OLS & $\mathrm{BE}$ \\
\hline
\end{tabular}

Note: the standard deviation is in parentheses; ${ }^{* * *},{ }^{* *},{ }^{*}$ are significant at the level of $1 \%, 5 \%$, and $10 \%$, respectively.

that this paper is more appropriate in the selection of parameters and the selection of model SEPDM. The overall satisfaction of the estimated value is significant at the level of $1 \%$, and only a few of the estimated values are not satisfied. Moreover, the whole result shows that in the process of gradually increasing variables, the symbols of the estimated values are consistent as a whole, and the variation range is very small. It is proved that the spatial error model selected in this paper conforms to a certain standard of regression, and the regression results are relatively reliable.

Model one estimates and analyzes the industrial structure represented by the proportion of the tertiary industry in the GDP separately. Comparing model two to model five, it is concluded that the industrial structure is an important factor affecting the spatial distribution of population in the Guangdong-Hong Kong-Macao Greater Bay area, and the impact is positive. The sign of the estimated income level is also positive, and it also performs well in the significant level test like the industrial structure. From this, we can see that the change of income level and industrial structure has promoted the spatial aggregation of population in the Guangdong-Hong Kong-Macao Greater Bay area.

Model 3 and model 5, the impact of traffic convenience and medical service level on people's life is self-evident. Most people have to carefully consider it when choosing work place and living place, because it has a huge impact on people's life and development. The model estimates in this paper also prove that the signs are all positive, and the significant level is basically $1 \%$. Therefore, the 
high level of transportation convenience and medical service will promote the population to gather in Guangdong-Hong Kong-Macao Greater Bay area.

The estimation results of basic education quality parameters shown in model 4 are a little unexpected. The estimation results of parameters are negative and significant at the level of $1 \%$. However, this result is also in line with the views of some statisticians and scholars in recent years, such as the difficulty of basic education enrollment in Shanghai in 2018. The number of school-age educated children in developed areas is increasing, but the number of primary schools and junior high schools has not changed much. Education investment mainly focuses on higher education. The number and scale of undergraduate colleges and universities are growing rapidly. Many school-age children choose to go back to their registered permanent residence. Although the state has constantly issued policies to solve the problem of education for the children of the migrant population, the problem of education for the migrant population still needs to be paid attention to in terms of the enrollment policy from the nearest school and the cost of education. If the problem of education for the children of migrant population cannot be solved, some parents will go back to the place of household registration together with their children, which has even affected the attitude of the new generation of young people living in big cities towards marriage and childbirth. Therefore, the quality of basic education in Guangdong, Hong Kong, Macao Greater Bay area has a certain degree of exclusion to the migrant population.

Model 6 introduces the housing price level into the data for estimation, which shows that the estimated result is positive, and the estimated value is significant at the level of 5\%. It is an indisputable fact that the higher the housing price is, the more the corresponding population in the region is. However, it cannot be said that the high housing price is the cause of population aggregation, but the high housing price in China represents the more perfect local infrastructure and public service facilities. At the same time, it can also be seen that although the high house price brings more pressure on people's life cost, it does not constitute the pressure of population aggregation. Compared with a single cost decision, people are more inclined to consider more cost-effective decision-making. It can also be seen from this that we cannot simply think that high house prices are bad, and we should comprehensively consider the multi-layer factors behind them.

\subsection{Result Discussion}

From the estimation results of model 1 to model 6, we can see that the symbols and statistical significance of the variables introduced in the model have not changed significantly, the only change is the size of the parameter estimation, and the range of change is not significant. This fully shows that under the condition of considering the existence of spatial effects, the above factors have a real impact on the spatial distribution of population in Guangdong-Hong Kong-Macao Great Bay area. 
In order to test the robustness of the model setting form and its parameter estimates, the following comparative analysis is made on the estimation results under different model setting forms. First, by comparing the estimation results of model 6, model 7 and model 8 It can be seen that the goodness of fit of models 7 and 8 is significantly lower than that of model 6, indicating that the estimation effect of the SEPDM is better than that of POI_OLS and ordinary BE methods.

\section{Conclusion}

This paper makes a series of spatial analysis on the data of Guangdong-Hong Kong-Macao Greater Bay area from 2005 to 2017, aiming to analyze the spatial aggregation characteristics and related trends of population in Guangdong-Hong Kong-Macao Greater Bay area, and to explore the influencing factors of spatial distribution of population in Guangdong-Hong Kong-Macao Greater Bay area. The conclusions are as follows: 1) Guangdong-Hong Kong-Macao Greater Bay area has a strong population attraction, the trend of population aggregation is still obvious, and the population of Guangdong-Hong Kong-Macao Greater Bay area is increasing. According to the relative migration index calculated in this paper, Guangzhou, Foshan, Shenzhen and Zhongshan belong to the relative migration area, and the population aggregation trend is relatively stronger. In terms of population geographical concentration, Hong Kong, Macao and Shenzhen have relatively high population density. Within the Guangdong-Hong Kong-Macao Greater Bay area, Shenzhen is the only city both in the first-class population density area and in the relative immigration area. 2) Using the spatial error model and the way of increasing variables gradually, this paper analyzes the influence of the parameters selected in this paper on the population distribution of Guangdong, Hong Kong and Macao Greater Bay area. The estimation results have passed the significant level test, so the estimation results are relatively stable. The results show that income level, industrial structure, medical condition and traffic convenience level all promote the population agglomeration of Guangdong, Hong Kong and Macao, while the quality of basic education hinders it. It can also be seen from the data that a certain degree of high housing prices has not had a negative impact on population agglomeration.

Suggestions: first, in order to further promote the overall urbanization process of Guangdong-Hong Kong-Macao Greater Bay area, it is necessary to speed up the adjustment of industrial structure, faster and better coordinated development, and attract more people to join; second, it is necessary to speed up the infrastructure and public service construction of Guangdong-Hong Kong-Macao Greater Bay area, and improve the functional level of urban services for people, especially transportation and other important aspects; third, it is necessary to make special mention. It is the problem of basic education. It is necessary to fully solve the systematic problem of insufficient investment and uneven distribution in basic education. Improving the level of basic education service must be 
conducive to the population agglomeration and urbanization development of Guangdong-Hong Kong-Macao Greater Bay area.

\section{Conflicts of Interest}

The author declares no conflicts of interest regarding the publication of this paper.

\section{References}

Deng, C. X., Li, M., \& Bin, J. Y. (2017). Spatial-Temporal Variation Characteristics and Main Influence Factors Analysis of Population Distribution Pattern in Hunan Province. Economic Geography, 37, 41-48.

Du, C. M., Jiao, L. M., \& Xu, G. (2018). Spatio-Temporal Patterns and Driving Factors of Urban Population Density Change of Prefecture-Level Cities in China during 2006-2016. Tropical Geography, 38, 791-798.

Du, Z. W., Zhang, H. O., Ye, Y. Y., Jin, L. X., \& Wang, C. J. (2019). Spatiotemporal Evolution and Influences of Urban Population Shrinkage in Guangdong Since 2000. Tropical Geography, 39, 20-28.

Liu, J., \& Tian, Y. S. (2018). Mutual Influencing Mechanism of Industry-Population-Space in the Pearl River Delta Urban Agglomeration in the Context of the Guangdong-Hong Kong-Macao Greater Bay Area development. Progress in Geography, 37, 1653-1662.

Liu, N. Q., \& Geng, W. C. (2015). On Evolution of Population Spatial Pattern in Shanghai and Its Influencing Factors: Empirical Research Based on Spatial Panel Model. Journal of Finance and Economics, 41, 99-110.

Meng, Z. M., \& Pan, X. (2018). The Influence of the Allocation of Basic Education Resources on the Spatial Evolution of Population in Shanghai. Urban Problems, No. 5, $34-45$.

Mu, Y. F. (2014). Research on Pattern Evolution of Employed Population in Core Zone of Yangtze River Delta. Geography and Geo-Information Science, 30, 70-75.

Shen, Y. C., \& Luo, T. Y. (2019). The Influence of Industrial Layout on the Spatial Distribution of Population and Suggestions for its Guidance in Beijing, Tianjin and Hebei. Review of Industrial Economics, No. 3, 21-36.

Wang, G. X., \& Qin, Z. Q. (2013). The Evolution of Spatial Relationship Between Population and Economy in Shanxi Province. Economic Geography, 33, 29-35. https://doi.org/10.1155/2013/195635

You, Z., Wang, L., Feng, Z. M., \& Yang, Y. Z. (2013). Spatio-Temporal Pattern and Variation Characteristics of Population Distribution at County Level in the Pearl River Delta. Tropical Geography, 33, 156-163.

Yu, R. L., Liu, C. L., \& Yang, Z. (2012). Spatio-Temporal Pattern Characters of Population Distribution in Wuhan Metropolitan Area. Resources and Environment in the Yangtze Basin, 21, 1087-1092.

Yu, T. T., Song, Y. X., Hao, F. L., \& Rong, A. (2017). Space Pattern Evolution of Population Distribution and Space Pattern Evolution of Population Distribution and the Driving Factors in Northeast China the Driving Factors in Northeast China. Scientia Geographica Sinica, 37, 709-717.

Zhou, Y., Tu, J. J., Lu, D. B., Yan, X., \& Wu, J. T. (2011). Study on the Relationship between Population and Economic Spatial Distribution and Its Dynamic in Chongqing. Economic Geography, 31, 1781-1785. 